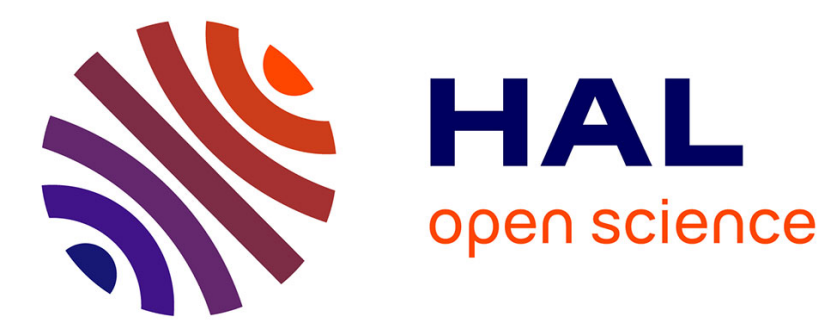

\title{
Popular Matching Games for Correlation-Aware Resource Allocation in the Internet of Things
}

Kenza Hamidouche, Walid Saad, Merouane Debbah

\section{To cite this version:}

Kenza Hamidouche, Walid Saad, Merouane Debbah. Popular Matching Games for Correlation-Aware Resource Allocation in the Internet of Things. GLOBECOM 2017 - 2017 IEEE Global Communications Conference, Dec 2017, Singapour, Singapore. hal-01986195

\section{HAL Id: hal-01986195 \\ https://hal-centralesupelec.archives-ouvertes.fr/hal-01986195}

Submitted on 18 Jan 2019

HAL is a multi-disciplinary open access archive for the deposit and dissemination of scientific research documents, whether they are published or not. The documents may come from teaching and research institutions in France or abroad, or from public or private research centers.
L'archive ouverte pluridisciplinaire HAL, est destinée au dépôt et à la diffusion de documents scientifiques de niveau recherche, publiés ou non, émanant des établissements d'enseignement et de recherche français ou étrangers, des laboratoires publics ou privés. 


\title{
Popular Matching Games for Correlation-aware Resource Allocation in the Internet of Things
}

\author{
Kenza Hamidouche ${ }^{1}$, Walid Saad ${ }^{2}$, and Mérouane Debbah ${ }^{1,3}$ \\ ${ }^{1}$ CentraleSupélec, Université Paris-Saclay, Gif-sur-Yvette,France, Email: kenza.hamidouche@ @entralesupelec.fr \\ ${ }^{2}$ Wireless@VT, Bradley Department of Electrical and Computer Engineering, Virginia Tech, Blacksburg, VA, USA, Email: walids@ vt.edu \\ ${ }^{3}$ Mathematical and Algorithmic Sciences Lab, Huawei France R\&D, France, Email: merouane.debbah@huawei.com
}

\begin{abstract}
In this paper, the problem of cell association is studied in an Internet of things (IoT) system in which a set of devices deployed in a given area report ground information to a set of small base stations (SBSs) via uplink communication links. In this model, the key goal is to prevent multiple devices from reporting the same information to a given SBS by taking into account the spatial correlation between the IoT devices. In particular, the problem of correlation-aware cell association is formulated as a popular matching game in which the IoT devices are assigned to the SBSs to maximize the amount of information that is reported to the SBSs. To this end, the number of matched devices to every SBS must be maximized. For the formulated problem, a distributed two-level matching algorithm is proposed and the algorithm is proved to converge to a popular outcome. In that state, all the SBSs and devices prefer the matching that results from the proposed algorithm to any other possible matching. Simulation results show the performance of the proposed model.
\end{abstract}

Index Terms-Matching games, Internet of thing, popularity.

\section{INTRODUCTION}

The number of Internet-connected devices has exponentially increased during the last decade and is expected to reach 50 billion devices by 2025 , giving rise to the Internet of things (IoT) ecosystem [1]. In particular, IoT will be composed of human type devices and machine types devices with heterogeneous quality-of-service (QoS) requirements depending on the applications. In fact, IoT offers a wide range of technologies that will revolutionize multiple sectors from homes security, automation and wearables to health care and automotive. To support all the traffic generated by the thousand of smart devices, next-generation cellular networks will need to accommodate the IoT and account for the new emerging challenges driven by the plethora of IoT devices [2,3]. Cellular networks will need to support novel traffic types that will typically consist of short files while also catering for new performance metrics that are more suitable for IoT systems such as reliability, latency and energy efficiency $[4,5]$.

One of the key challenges in IoT systems is cell association via which the IoT devices are assigned to SBSs to report the data they collect. Although cell association is a well investigated problem in traditional cellular networks, new challenges emerge in the presence of IoT devices. For instance, the classical objectives such as interference mitigation, traffic

This research was supported by the ERC-PoC 727682 CacheMire project, the U.S. Office of Naval Research (ONR) under Grant N00014-15-1-2709 and the U.S. National Science Foundation under Grants CNS-1513697 and CNS1460316 offloading, and capacity maximization must evolve to account for the specific characteristics of IoT systems. In fact, in IoT systems, it is more important to account for the high density of the devices that are deployed, system reliability, and autonomy of the devices. This is induced by the short data size that are transmitted by the IoT devices which is different from classical user devices' traffic that consist mainly of streaming content, transmitted via downlink communication links. Moreover, the IoT systems must be designed to autonomously manage their data transmission and optimize their energy consumption. To cope with the energy and processing constraints at the IoT devices, the SBSs collect information about the physical events from multiple IoT devices. In addition to the collaborative nature of IoT systems that brings significant advantages over traditional transmission and sensing mechanisms, correlation between the deployed IoT devices is another characteristic of IoT systems that should be exploited to further improve the performance of the networks. In fact, the correlation among the observations of multiple IoT devices deployed in a same area can be exploited to limit the number of devices that might report the same information to a given small base station (SBS). Thus, the allocation of network resources including energy and spectrum can be designed more efficiently.

Several works were proposed in the literature to develop novel self-organizing and energy-efficient mechanisms that are more suitable for IoT systems. In [3], the authors provided a survey on the technical challenges posed by IoT devices and introduced different techniques for overcoming these challenges. The economics of IoT systems are analyzed in [6] with a focus on information pricing and its value. The work in [7] described the importance of self-organization in the IoT including several operations that should be performed by the IoT devices in a distributed and autonomous manner such as neighbor discovery, medium access control, energy management, and local connectivity. The authors in [8] proposed a novel approach for the deployment of unmanned aerial vehicles (UAVs) to collect data from the IoT devices. To ensure energy-efficient uplink communications, the IoT devices are clustered into multiple sets and then each cluster is assigned a single UAV to which the IoT devices can send their collected data. The work in [9] addressed the problem of distributed uplink random access in IoT systems. The problem was formulated as a noncooperative game in which the devices are the players that aim to meet their QoS requirements.

Remarkably, none of these works has accounted for the 
association of IoT devices to the SBSs or captured the correlation among the observations of the IoT devices as well as the the impact of short data packets on the system model. The main contribution of this paper is to analyze devicecell association in an IoT system serviced by a wireless cellular network while explicitly accounting for the correlation between the data reported by the different IoT devices. In this model, the key goal is to develop a new self-organizing scheme that enables the IoT devices to select, in a distributed manner, the SBSs to which they report the data they collect. In particular, we formulate a popular two-sided matching game with externalities between the IoT devices and SBSs [10-12]. The devices aim to ensure a given correlation level with the other IoT devices that are assigned to the SBSs while the SBSs are interested in the reliability level with which each of the devices can report the sensed information. A two-level matching algorithm is proposed based on the classical deferred acceptance algorithm [10] to match the IoT devices to the SBSs. Moreover, we prove that the proposed algorithm is guaranteed to converge to a popular matching outcome. This result shows that the number of assigned IoT devices to every SBS is maximized and there does not exist another matching that is preferred by a higher number of players.

The rest of the paper is organized as follows. In Section II, we present the system model and formulate the problem. In Section III, the optimization problem is formulated as a popular matching game and a decentralized algorithm is proposed and analyzed. Section IV presents the simulation results and.... Finally, Section V concludes the paper.

\section{SySTEM MODEL}

Consider a wireless IoT system composed of a set $\mathcal{I}$ of $I$ IoT devices deployed within a geographical area. In this area, a set $\mathcal{S}$ of $S$ SBSs is deployed to collect the data from the IoT devices via uplink communications.

For multiple access, the $I$ devices adopt a random access protocol in order to transmit to the SBSs. In particular, each device $i \in \mathcal{I}$ sends $n_{i}$ bits to its assigned SBS. Each packet should be delivered within a limited time period that corresponds to $c_{i}$ channel uses. The devices use a CSMA/CA protocol in which each device picks randomly one of the $K$ slots in the frame to send its packet. If two or more devices transmit during the same slot, then a collision occurs and none of the packets is received correctly by the SBSs. In classical wireless networks, the achievable capacity is used as a metric to assess the performance of the network. However, such a metric is only valuable for devices that transmit packets of large size, which is not the case in IoT systems in which IoT devices are typically sensors or machine type devices that must ensure a reliable transmission of small packets [5]. Thus, every IoT device $i$ seeks to transmit its small size packets while maintaining its packet error probability lower than a predefined threshold $\epsilon_{i}$ depending on the reliability level that is required for transmitting the considered packet. The probability of having an $\epsilon$-outage capacity equal to $\epsilon$ for a device $i$ that transmits its traffic to an SBS $s$ is given by:

$$
\operatorname{Pr}\left\{c_{i s}(\epsilon)>w \log \left(1+\frac{\left|h_{i s}\right|^{2} p_{i s}}{\sigma^{2}}\right)\right\}=\epsilon,
$$

where $w$ is the bandwidth, $p_{i s}$ is the transmit power from device $i$ to SBS $s, \sigma^{2}$ is the variance of the additive white Gaussian noise, and $h_{i s} \approx \mathcal{C N}(0,1)$ is the channel gain between device $i$ and SBS $s$. We assume the channels to be independent block Rayleigh fading and for $\epsilon \ll 1$, the $\epsilon$-outage capacity for a device $i$ that transmits its traffic to an SBS $s$ is given by:

$$
c_{i s}(\epsilon)=w \log \left(1+\frac{\epsilon p_{i s}}{\sigma^{2}}\right),
$$

Given a packet of size $n_{i s}$ and a packet error threshold $\epsilon$, the required rate by device $i$ when served by $\operatorname{SBS} s$ is given by [4]:

$$
r_{i s}\left(n_{i s}, \epsilon\right)=c_{i s}(\epsilon)+\frac{\log \left(n_{i s}\right)}{2 n_{i s}} .
$$

Thus, the maximal achievable rate $r_{i s}\left(n_{i s}\right)$ should satisfy the condition $r_{i s}\left(n_{i s}, \epsilon\right) \leq r_{i s}\left(n_{i s}\right)$, where $r_{i s}\left(n_{i s}\right)$ is given by:

$$
r_{i s}=w \log \left(1+\frac{\left|h_{i s}\right|^{2} p_{i s}}{\sigma^{2}}\right)+\frac{\log \left(n_{i s}\right)}{2 n_{i s}} .
$$

Due to the high density of the devices deployed to report information on the ground, multiple IoT devices might report the same information to a given SBS $s$. For instance, typical wireless sensor applications require a dense deployment of sensors to achieve satisfactory coverage [13]. As a result, multiple IoT devices record information about the same event which increases the degree of correlation among the observations of the devices. Ignoring such correlation may lead to an inefficient allocation of the frequency bands that might be allocated to multiple IoT devices to send the same information to a given SBS. Moreover, as the number of devices that the SBSs can support simultaneously is limited, the transmission of critical and urgent information to an SBS might be delayed due to inefficient cell association policy. Next, we model the correlation between the information reported by different devices.

\section{A. Data Correlation Model}

Each device $i$ is assumed to report an estimation $\widehat{D}_{i}$ of a source information $D_{i}$, which might be correlated to the information reported by other devices that are deployed in the same area. Once a device observes an information, it encodes the information into a signal $Y_{i}$ and then transmits the information to its assigned SBS. Each observed information $\widehat{D}_{i}$ by a device $i$ can be written as:

$$
\widehat{D}_{i}=D_{i}+N_{i}
$$

where $N_{i}$ is the observation noise which is assumed to be a Gaussian random variable. Thus, $\widehat{D}_{i}$ is also a Gaussian random variable of mean $\mu_{i}$ and variance $\sigma_{i}^{2}$. Moreover, the noise at each device is independent from the noise at the other devices, i.e., $N_{i}$ and $N_{j}$ are independent for all $i \neq j \in \mathcal{I}$. Given the 
observation $\widehat{D}_{i}$, the encoded version that device $i$ transmits to the SBS can be written as:

$$
Y_{i}=f_{i}\left(\widehat{D}_{i}\right) .
$$

When an SBS receives the information, it decodes the data and reconstruct an estimate of the source information.

Assume that the observations $\widehat{\mathcal{D}}=\left\{\widehat{D}_{1}, \ldots, \widehat{D}_{I}\right\}$ collected by all the devices follow a multivariate Gaussian distribution. To model the correlation between the observations reported by two devices, we use the power exponential model as the physical event information such as electromagnetic waves, is modeled to have an exponential autocorrelation function [14]. Hence, the covariance $\phi_{i j}$ between devices $i$ and $j$ separated in distance by $d_{i, j}$ is given by [15]:

$$
\phi_{i j}=\operatorname{cov}\left(\widehat{D}_{i}, \widehat{D}_{j}\right)=e^{\left(\frac{-d_{i j}}{\kappa}\right)^{\delta}},
$$

where $d_{i j}=\left\|\boldsymbol{z}_{i}-\boldsymbol{z}_{j}\right\|$ is the distance between device $i$ and device $j$ located at coordinates $\boldsymbol{z}_{i}$ and $\boldsymbol{z}_{j}$, respectively. $\delta, \kappa>0$ are constant that show the impact of the distance variation between two devices on the data correlation. We set $\delta=2$ and adjust the correlation factor $\kappa$ to account for systems with different levels of data correlation. The covariance is assumed to be nonnegative and decreases monotonically with the distance $d_{i, j}$. When the distance between the two devices increases, the value of the covariance approaches 1 and approaches 0 when the distance goes to $\infty$.

This model captures the spatial correlation between the devices and will be used next to achieve an efficient allocation of network resources and prevent the SBSs from receiving redundant information.

\section{B. Problem Formulation}

In IoT systems, the density of connected IoT devices may not be uniform. Thus, the usage of classical received signal strength indication (RSSI)-based cell association methods might lead some cells to serve a higher number of IoT devices compared to other cells resulting in load imbalance between the SBSs. Moreover, intuitively, receiving data from spatially separated IoT devices is more useful for the SBSs than highly correlated data from closely located devices. Hence, it may not be necessary for every device to transmit its data to the same SBS, instead, a smaller number of devices' measurements might be adequate to communicate an event feature to a single SBS. In addition, such redundancy induces inefficient usage of energy at both SBSs and IoT devices as well as network resources that are allocated to the devices and SBSs to transmit redundant information.

To develop efficient cell association schemes for IoT systems, one should account for the correlation between the content that is reported by multiple devices. The device-cell assignment problem can be represented by a decision policy $\mu$ which for any device-SBS pair $(i, s) \in \mathcal{I} \times \mathcal{S}$, it outputs a binary variable $\alpha_{i s} \in\{0,1\}$, where $\alpha_{i s}=1$ indicates that device $i$ reports its collected information to SBS $s$ and $\alpha_{i s}=0$, otherwise. Moreover, we define the load of an SBS $s$ as follows:

$$
\eta_{s}= \begin{cases}\sum_{i=1}^{I} \alpha_{i s} \frac{\widehat{D}_{i}}{r_{i s}\left(n_{i s}\right)}, & \text { if } r_{i s}\left(n_{i s}, \epsilon\right) \leq r_{i s}\left(n_{i s}\right), \\ 0, & \text { otherwise. }\end{cases}
$$

which represents the fraction of time that is required for all the devices assigned to an SBS $s$ to transmit their data to the SBS with their required packet probability error.

The assignment of the devices to the SBSs as well as the allocation of the network resources have an impact on the performance of the IoT system. The goal of each SBS is to get the information from the devices and be able to decode it successfully. However, using conventional cell association approaches such as max-SINR and max-RSSI schemes in highly dense networks might result in ineffective reports of information to the SBSs. In fact, in such schemes, the devices report their data to the SBSs without accounting for the correlation of their transmitted information with the data reported by other devices to the same SBSs. Moreover, such metrics are effective when devices transmit packets of large size which is not the case in IoT systems. Instead, in an IoT system, the key goal is to maximize the number of useful bits that are received by each SBS. A data $\widehat{D}_{i}$ reported by a device $i$ to an SBS $s$ is considered useful if the correlation level between the considered device $i$ and other devices that are assigned to the same SBS $s$ is below a given predefined threshold $\theta_{s}$ which might differ from a given SBS to another. However, the SBSs need to receive sufficient data on a given parameter from the devices to ensure the correctness of the information reported by the different devices.

Assume that there is a number of $X$ parameters in the set $\mathcal{X}=\left\{x_{1}, \ldots, x_{X}\right\}$ on which each SBS wants to get information from the devices. We denote the set of devices that are associated to an SBS $s$ by $\mathcal{I}_{s} \subseteq \mathcal{S}$. We introduce the notion of set correlation factor for a given device $i$ and a set $\mathcal{I}_{s}$ as the correlation factor between device $i$ and device $j \in \mathcal{I}_{s}$ with which it achieve the highest correlation value given all the devices in $\mathcal{I}_{s}$, which is given by:

$$
\Phi_{i \mathcal{I}_{s}}(\mu)=\left\{\phi_{i \hat{j}} \mid \max _{\hat{j}} \phi_{i j}, \forall j \in \mathcal{I}_{s}(\mu)\right\},
$$

where $\mathcal{I}_{s}$ depends on the allocation $\mu$ as it represents the set of devices assigned to each SBS given by the vector $\alpha$. Thus, we have $\alpha_{i s}=1$ for all $i \in \mu(s) \subseteq \mathcal{I}$.

The device-SBS assignment problem in an IoT system can be formulated as follows:

$$
\begin{array}{cl}
\underset{\boldsymbol{\alpha}}{\operatorname{minimize}} & \sum_{s \in \mathcal{S}} \sum_{i \in \mathcal{I}} \alpha_{i s} \Phi_{i \mathcal{I}_{s}}(\mu), \\
\text { subject to } & \sum_{s \in \mathcal{S}} \alpha_{i s} \leq 1, \forall i \in \mathcal{I}, \\
& \eta_{s} \leq \eta_{s}^{\text {th }}, \forall s \in \mathcal{S}, \\
& p_{i s} \alpha_{i s} \leq p^{\text {th }}, \forall(i, s) \in \mathcal{I} \times \mathcal{S}, \\
& \mathcal{I}_{x s} \leq s_{x}, \forall s \in \mathcal{S} \text { and } x \in \mathcal{X}, \\
& \alpha_{i s} \in\{0,1\},
\end{array}
$$


where $\eta_{s}^{\text {th }}$ is the maximum traffic load that can be supported by $\operatorname{SBS} s, p^{\text {th }}$ is the probability threshold for receiving packets successfully, and $\mathcal{I}_{x s}$ is the set of devices that report information about parameter $x$ to SBS $s$. The goal is to minimize the sum of the correlation factors between the devices that are assigned to the same SBS. The first constraint ensures that one device is assigned to a single SBS. The second and third constraints ensure that the devices assigned to an SBS have the minimum required probability for successful transmission allowing the SBSs to decode the transmitted data without exceeding their capacity. The fourth condition ensures that the number of devices that report the same information to the same SBS should be limited.

\section{GAME FORMULATION AND ANALYSIS}

The uplink association problem in (10) is an NP-hard problem which does not admit a closed-form solution and has exponential complexity. In such problems, centralized cell association schemes require the SBSs to send the network information to radio network controller.

\section{A. Cell Association as a Matching Game with Externalities: Preliminaries}

To solve the problem in (10), we propose a novel solution based on matching theory, a mathematical framework that provides decentralized solution with tractable complexity for combinatorial problems such as the one in (10) [16]. A twosided matching game is an assignment problem between two disjoint sets of players in which the players of each set have preferences over the players of the other set. In our context, the matching is between the two sets of devices $\mathcal{I}$ and SBSs $\mathcal{S}$. Each player ranks the players of the opposite set based on a preference relation that allows every player to decide which SBS/IoT device can enable it to maximize its own benefit in the system.

To define the preference relations, we introduce individual utility functions for each device and SBS, using which they can rank one another. In the proposed cell association problem, the preference relation of the SBSs will depend only on the packet error probability while the devices will transmit their information based on its set correlation factor with the devices that are willing to be assigned to each of the SBSs. Thus, the preferences of the devices not only depend on the SBSs but also on the current matching which introduces externalities in the matching problem. Such problem corresponds to the problem of two-sided matching in which hospitals are matched to residents who have strict preferences over the hospitals based on the residents that are assigned to each hospital. In our model, hospitals correspond to the SBSs while the residents are the devices that determine their preferences over SBSs depending on the devices assigned to each SBS.

For the game we consider $\mathcal{G}=\left(\mathcal{S} \cup \mathcal{I}, \succ_{i}, \succ_{s}\right)$, the preference relation $\succ$ is defined as a complete, reflexive, and transitive binary relation between the elements of a given set. We denote the preference relation of each SBS $s$ over the set $\Upsilon(\mathcal{I})$ of subsets of $\mathcal{I}$, by $\succ_{s}$ and use $\mathcal{I} \succ_{s} \mathcal{I}^{\prime}$ when SBS $s$ prefers to receive information from the subset $\mathcal{I}$ of devices than receiving it from the subset $\mathcal{I}^{\prime}$. An SBS is assumed to be matched to $i_{0}$ to represent the case in which an SBS prefers not to receive any information from the devices. Similarly, $\succ_{i}$ denotes that the preference relation of a device $i \in \mathcal{I}$ over the sets $\left(\mathcal{S} \times \Upsilon(\mathcal{I})_{i}\right) \cup\{(\emptyset, \emptyset)\}$, where $\Upsilon(\mathcal{I})_{i}=\left\{\mathcal{I}^{\prime} \in \Upsilon(I): i \in \mathcal{I}^{\prime}\right\}$.

\section{B. Cell Association as a Matching Game with Externalities}

Each cell association policy $\mu$ determines the set of devices that are assigned to each SBS. Thus, the problem can be defined as a one-to-many matching game with externalities:

Definition 1. Given two disjoint sets of SBSs and devices $\mathcal{S}$ and $\mathcal{I}$, the cell association policy, $\mu$, can be defined as a mapping, $\mu: \mathcal{S} \cup \mathcal{I} \rightarrow \mathcal{I} \cup \mathcal{I} \times \mathcal{S}$ that satisfies:

1) $\forall s \in \mathcal{S}, \mu(s) \in \Upsilon(\mathcal{I})$,

2) $\forall i \in \mathcal{I}, \mu(i) \in\left(\mathcal{S} \times \Upsilon(\mathcal{I})_{i}\right)$,

3) $\mu(i)=(s, \mu(s))$ whenever $i \in \mu(s)$, and

4) $\mu(s)=\mathcal{I}^{\prime}$ if and only if $\left(s, \mathcal{I}^{\prime}\right)=\mu(i)$ from some $i \in \mathcal{I}$.

$\mu(s)=\mathcal{I}^{\prime}$ implies that $\alpha_{i s}=1, \forall i \in \mathcal{I}^{\prime}$, otherwise, $\alpha_{i s}=0$. Form Definition 1, we can deduce that the first and third conditions in (10) are satisfied. Moreover, the matching is feasible, if it satisfies the capacity constrains of the SBSs which is defined as $\eta_{s} \leq \eta_{s}^{\text {th }}, \forall s \in \mathcal{S}$. In contrast to classical one-tomany matching games in which the players of one side are only matched to the players of the other set, in the defined matching game, the devices are also matched to other devices in addition to the SBSs. Next, we define suitable utility functions for the two sets of players.

\section{Utility and Preference Relations of the Devices and SBSs}

The devices have preferences over both SBSs and devices. The preference relation of a given device depends on both SBS and devices that are assigned to the considered SBS. Such preference relations called nonclassical preference relation, are different from the preference relation in classical one-to-many matching games in which the devices only have preferences over the SBSs. The devices build their preferences based on the achievable rate and its set correlation factor with the devices matched to each SBS. Thus, we define the utility of device $i$ when matched to the tuple $(s, \mu(s))$ as:

$$
U_{i}(s, \mu)=\omega_{i} r_{i s}\left(n_{i s}, \epsilon_{i}\right)+\left(1-\omega_{i}\right) \Phi_{i \mathcal{I}_{s}(\mu)},
$$

where $\omega_{i}$ is a weighting parameter that allows the device to control the impact of the correlation factor on the overall decision of the device. Thus, the device can balance between the achievable rate and the correlation factor between device $i$ and the devices assigned to SBS $s$. In fact, the second term in (11) enables a device $i$ to report its information to an SBS that cannot get the same information from its assigned devices. Using the utility in (11), the preference relations of the devices can be given by:

$$
(s, \mu(s)) \succ_{i}\left(s^{\prime}, \mu\left(s^{\prime}\right)\right) \Longleftrightarrow U_{i}(s, \mu)>U_{i}\left(s^{\prime}, \mu\right),
$$


for $\forall i \in \mathcal{I}$, and $\forall s, s^{\prime} \in \mathcal{S}$

To determine which devices will be assigned to each SBS, the SBSs define a preference relation based on the $\epsilon$-outage capacity that can be ensured by each of the device. In other words, the higher the $\epsilon$-outage capacity of a device, the more preferred is the device to the SBS. The preference relations of the SBSs can be given as follows:

$$
i \succ_{s} i^{\prime} \Longleftrightarrow r_{i s}\left(n_{i s}, \epsilon_{i}\right)>r_{i^{\prime} s}\left(n_{i s}, \epsilon_{i}\right) \text {. }
$$

Next, we propose a novel matching algorithm that matches the devices to the SBSs while accounting for the correlation between the data reported by the devices and the induced externalities.

\section{Proposed Correlation-Aware Cell Association Algorithm}

The desirable outcome in a two-sided matching problem is a stable matching state in which every two players that prefer one another to any other player should be matched. To reach the stable outcome in one-to-many matching games, the deferred acceptance algorithm was proposed in [17]. However, in [17], the rural hospital theorem states that using the deferred acceptance algorithm, the SBSs are always matched to the same number of devices under any stable matching.

Thus, even though stability of a matching guarantees that every SBS and device that prefer one another over other players are matched, it does not guarantee that the SBSs will reach their capacity and be matched to a number of IoT devices that they can support. This is important in IoT systems as the SBSs need the receive the information from multiple devices to ensure the reliability of the received data. Moreover, when the IoT devices report information about multiple parameters, the SBSs must receive the information quickly and about all these parameters. These information is then used to take actions which might be critical for delay-sensitive applications.

Thus, stability in the formulated game is not desirable by the players as an SBS would prefer to be matched with the maximum number of devices so that it can get all the required information from the devices deployed in the area it covers. To this end, we relax the condition of local stability of the matching to the popularity of a matching which corresponds to the global stability. Given two matchings $\mu$ and $\mu^{\prime}$, matching $\mu$ is more popular than matching $\mu^{\prime}$ if the number of players that prefer matching $\mu$ is higher than the number of players that prefer matching $\mu^{\prime}$. In other words, a matching $\mu$ is the most popular matching if there does not exist another matching that is preferred by more players as compared to the matching $\mu$.

To define the most popular matching $[11,12]$, we introduce the notion of a vote that allows every SBS and device to vote for its preferred matching. For a device $i$, the vote function $\mathrm{v}_{i}\left(\mu(s) \cup\{s\}, \mu\left(s^{\prime}\right) \cup\left\{s^{\prime}\right\}\right)$ equals 1 if $(s, \mu(s)) \succ_{i}\left(s^{\prime}, \mu\left(s^{\prime}\right)\right)$ which means that device $i$ prefers SBS $s$ that is matched to a set of devices $\mu(s)$ to SBS $s^{\prime}$ that is matched to a set of devices $\mu\left(s^{\prime}\right)$. The vote function equals -1 if $\left(s^{\prime}, \mu\left(s^{\prime}\right)\right) \succ_{i}(s, \mu(s))$ which means that device $i$ prefers $\operatorname{SBS} s^{\prime}$ that is matched to a set of devices $\mu\left(s^{\prime}\right)$ to SBS $s$ that is matched to a set of devices $\mu(s)$, and it is 0 if device $i$ is indifferent between the two SBSs. Thus, for two matchings $\mu$ and $\mu^{\prime}$, the preference of a device $i$ can be redefined as follows:

$$
\Delta_{i}\left(\mu, \mu^{\prime}\right)=\mathrm{v}_{i}\left(\mu(i), \mu^{\prime}(i)\right) .
$$

In (13), we defined the preferences of the SBSs over individual devices. Having two different sets of devices $\mathcal{I}_{1} \subseteq \mathcal{I}$ and $\mathcal{I}_{2} \subseteq \mathcal{I}$, the SBS should be able to determine its preference while capturing its capacity and the required capacity for serving the devices in a given set. To this end, we assume that an SBS can determine the mean required capacity for serving a packet based on its history. Thus, given a set $\mathcal{I}_{1}$ that contains $\left|\mathcal{I}_{1}\right|$ devices, we add to the set $\mathcal{I}_{1}, q_{s}-\left|\mathcal{I}_{1}\right|$ occurrences of the element $i_{0}$, where $q_{s}$ being the capacity of SBS $s$. This makes the two sets $\mathcal{I}_{1}$ and $\mathcal{I}_{2}$ of capacity $q_{s}$. Thus, we can define the sets $\widehat{\mathcal{I}}_{1}=\mathcal{I}_{1} \backslash \mathcal{I}_{2}$ and $\widehat{\mathcal{I}}_{2}=\mathcal{I}_{2} \backslash \mathcal{I}_{1}$ as a vector given by $\left[\widehat{\mathcal{I}_{1}}(1), \ldots \widehat{\mathcal{I}_{1}}(k)\right]$ and $\left[\widehat{\mathcal{I}_{2}}(1), \ldots \widehat{\mathcal{I}_{2}}(k)\right]$ for $k=q_{s}-\left|\mathcal{I}_{1} \cap \mathcal{I}_{2}\right|$. The preference of the SBS over the sets of devices can thus be given by:

$$
\delta_{s}\left(\mathcal{I}_{1}, \mathcal{I}_{2}\right)=\min _{\sigma \in \Pi|k|} \sum_{j=1}^{k} \mathrm{v}_{s}\left(\widehat{\mathcal{I}}_{1}(j), \widehat{\mathcal{I}}_{2}(\sigma(j))\right),
$$

where $\Pi[k]$ is the set of permutation on $\{1, \ldots, k\}$. Thus, we can define $\Delta_{s}\left(\mu, \mu^{\prime}\right)$ as $\delta_{s}\left(\mathcal{I}_{1}, \mathcal{I}_{2}\right)$. So $\Delta_{s}\left(\mu, \mu^{\prime}\right)$ counts the number of votes by SBS $s$ for $\mu(s)$ compared to $\mu^{\prime}(s)$ when the set $\widehat{\mathcal{I}}_{1}=\mu(s) \backslash \mu^{\prime}(s)$ and $\widehat{\mathcal{I}}_{2}=\mu(s) \backslash \mu^{\prime}(s)$.

Finally, two matchings $\mu$ and $\mu^{\prime}$ can be compared based on a function $\Delta\left(\mu, \mu^{\prime}\right)$ defined as follows:

$$
\Delta\left(\mu, \mu^{\prime}\right)=\sum_{j \in \mathcal{S} \cup \mathcal{I}_{1}} \Delta_{j}\left(\mu, \mu^{\prime}\right) .
$$

A matching $\mu$ is considered at least as popular as matching $\mu^{\prime}$ if $\sum_{j \in \mathcal{S} \cup \mathcal{I}_{1}} \Delta_{j}\left(\mu, \mu^{\prime}\right) \geq 0$ and $\mu$ is more popular than $\mu^{\prime}$ if $\sum_{j \in \mathcal{S} \cup \mathcal{I}_{1}} \Delta_{j}\left(\mu, \mu^{\prime}\right)>0$. Thus, a popular matching can be defined as follows.

Definition 2. A matching $\mu$ is a popular matching in the matching problem $\mathcal{G}=\left(\mathcal{S} \cup \mathcal{I}_{1}, \prec_{i}, \prec_{s}\right)$ if $\sum_{j \in \mathcal{S} \cup \mathcal{I}} \Delta_{j}\left(\mu, \mu^{\prime}\right) \geq 0$ for every matching $\mu^{\prime}$ in $\mathcal{G}$.

Now, we propose a distributed matching algorithm to reach a popular matching which is based on the classical deferred acceptance algorithm in [17]. In this regard, we consider a graph $G^{\prime}=\left(\mathcal{I}^{\prime} \cup \mathcal{S}, \mathcal{E}^{\prime}\right)$ defined as follows: $\mathcal{I}^{\prime}$ consists in a set of devices that contains two copies $i^{0}$ and $i^{1}$ of every device $i \in \mathcal{I}$. The set of SBSs $\mathcal{S}$ remains the same and the edge set $\mathcal{E}^{\prime}$ is defined by $\mathcal{E}^{\prime}=\left\{\left(i^{0}, s\right),\left(i^{1}, s\right):(i, s) \in \mathcal{E}\right\}$.

The preference list of $i^{0}$ and $i^{1}$ are the same preference list of device $i$. The devices in the set $\left\{i^{0}, i^{1}: i \in \mathcal{I}\right\}$ will be called bottom level or level 0 for $i^{0}$ and top level or level 1 for $i^{1}$. Every SBS $s$ prefers any level 1 device to a level 0 device within the set of devices. For instance, in a set composed of two devices $i_{1}, i_{2}$ and the initial preference of SBS $s$ given by $i_{1} \succ_{s}$ $i_{2}$, the new preference of SBS $s$ in the set $\mathcal{I}^{\prime}=\left\{i_{1}^{0}, i_{2}^{0}, i_{1}^{1}, i_{2}^{1}\right\}$ 
Table I: Proposed Context-Aware Assignment Algorithm

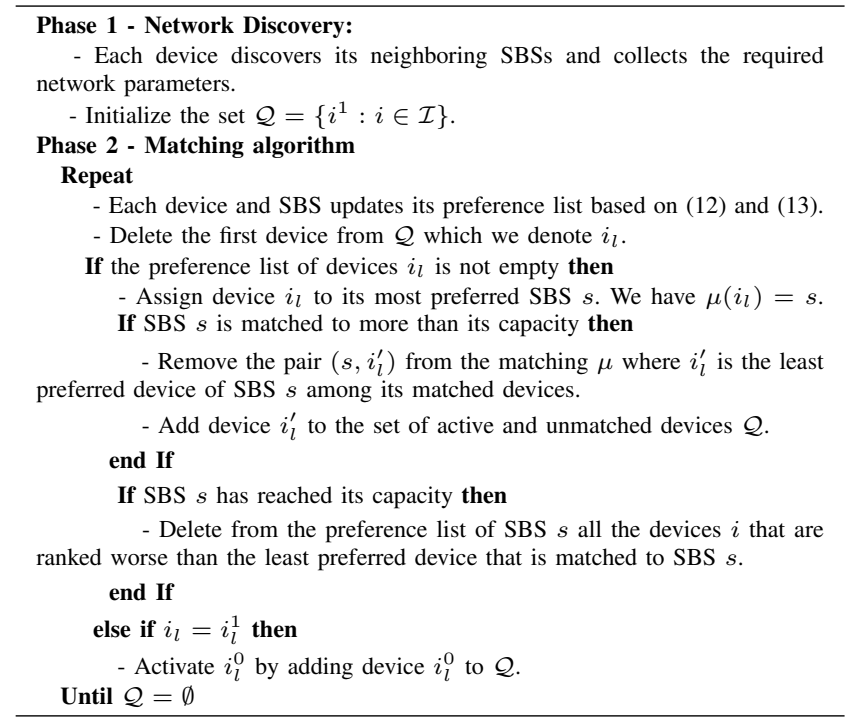

is given by $i_{1}^{1} \succ_{s} i_{2}^{1} \succ_{s} i_{1}^{0} \succ_{s} i_{2}^{0}$. Initially, all level 0 devices are assumed to be active while all level 1 devices are inactive. The idea of the two-level players is to promote the devices that are rejected by all the SBSs by removing these devices from the bottom level and only keeping the top level version of these devices which is more preferred by the SBSs given the new preference list of the SBSs that accounts for the level of the devices. Moreover, we introduce a queue $\mathcal{Q}$ that contains all the active and unmatched devices and then the deferred acceptance algorithm is applied as follows:

- Initially, the set of top level devices is empty and only the devices in the bottom level set are active and can propose to their most preferred SBSs.

- Every unmatched active device $i^{1}$ or $i^{0}$ proposes to its most preferred SBSs to which it has not proposed yet.

- In case an active device $i^{0}$ has been rejected by all the SBSs, the device is removed from the queue $\mathcal{Q}$ and the device $i^{1}$ becomes active and joins the queue $\mathcal{Q}$.

- When an SBS receives a request from a device $i^{1}$ or $i^{0}$, the SBS accept the requests based on its preference list. If the SBS is matched to more than its capacity, it reject the least preferred device that we denote $i^{\prime}$ and this device joins the queue of unmatched devices $\mathcal{Q}$.

For the algorithm we proposed, we can derive the following result regarding its convergence and popularity.

Theorem 1. The proposed matching algorithm with preferences over devices is guaranteed to converge to a max-sized popular matching.

Proof. The proof is provided in the Appendix.

\section{Simulation Results}

For our simulations, we consider that the IoT devices are deployed in an area that is covered by 20 SBSs. The transmit power of the IoT devices is $0.5 \mathrm{~W}$, the bandwidth is set to 10
$\mathrm{MHz}$, the noise to $-50 \mathrm{dBm}$, and $\epsilon=0.005$. The correlation model parameters are set as follows $\delta=1$ and $\kappa>0$.

To show the performance of the proposed algorithm for the assignment of IoT devices to the SBSs, we consider that there are 10 different events that are observed by the IoT device and every device can only observe one event. Then, we apply the proposed algorithm for every event and the capacity of the SBSs is considered as the minimum number of correlated IoT devices that are required by the SBSs to approach the real value of the sensed event. In our simulations, we assume that two IoT devices are correlated when the correlation level that exceeds 0.7 .

In Fig 1a, we compare the mean number of events among the 10 events that are reported successfully to the SBSs as a function of the total number of IoT devices that are deployed. The results of the proposed algorithm are compared with classical deferred acceptance algorithm that converges to a stable outcome. The mean number of events that are successfully reported to the SBSs increases with the number of IoT devices since the probability of correlation between the IoT devices that are assigned to the same SBS increases. Fig 1a shows that popularity of the cell association algorithm achieves substantial performance gains allowing the SBSs to detect more than 2 to 3 events over 10 events, compared to the classical stable cell association algorithm.

Fig 1b shows the percentage of useful information that is reported to the SBSs by the IoT devices. The proposed correlation-based algorithm is compared with the maximum sum-rate based deferred acceptance algorithm. In the later, neither the devices nor the SBSs account for the correlation between the information observed by the IoT devices and the SBSs aim to maximize the sum-rate of their assigned IoT devices. The results show that the amount of useful information is up to $40 \%$ higher in the proposed algorithm since the correlated devices are not assigned to the same SBSs after a given threshold that ensures that SBSs can approach the real statistics about the observed events.

In Fig 1c, we compare the proposed algorithm with the maximum sum-rate algorithm by showing the amount of useful information that is gathered by the SBSs as a function of the correlation parameter $\kappa$. Fig 1c shows that the percentage of useful information decreases by increasing the correlation level between the IoT devices. In fact, increasing the correlation level between the 250 IoT devices that are deployed increases the number of devices that report the same information to the same SBSs. However, we can see that the proposed algorithm outperforms the maximum sum-rate algorithm in which the cell association mechanism ignore the correlation level between the IoT devices since the redundant information at a given SBS is reduced by the proposed algorithm event though the number of devices is large which increase the probability of reporting the same observations to a given SBS. 


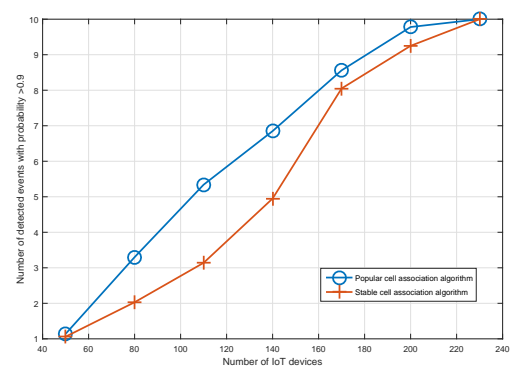

(a)

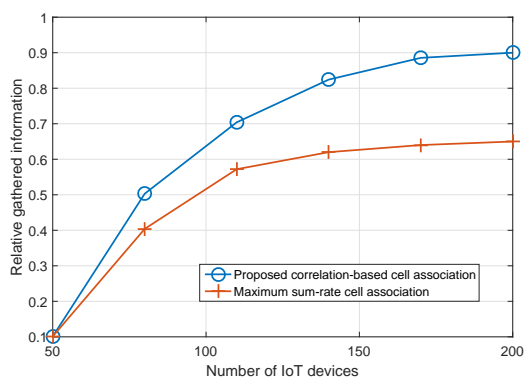

(b)

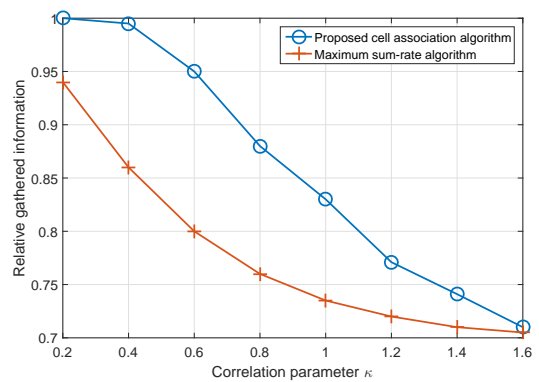

(c)

Figure 1: Numerical results for a) number of detected events successfully as a function of the number of IoT devices, b) useful gathered information by the SBSs as a function of the number of IoT devices, and c) useful gathered information by the SBSs as a function of the correlation parameter $\kappa$.

\section{Conclusion}

In this paper, we have proposed a novel framework of cell association for IoT-driven SCNs. We have formulated the problem of cell association as a two-sided matching problem between the two sets of devices and SBSs. To maximize the number of devices that are matched to each SBS, we have relaxed the stability solution concept into popularity and proposed a distributed matching algorithm that is guaranteed to converge to a popular outcome. Moreover, we have considered the correlation between the different IoT devices that are deployed in a given area to improve the efficiency of the cell association mechanism. Simulation results have shown that the proposed algorithm outperforms the classical cell-association mechanisms.

\section{REFERENCES}

[1] D. Evans, "The internet of things: How the next evolution of the internet is changing everything," Cisco, White paper, 2011.

[2] L. Atzori, A. Iera, and G. Morabito, "The internet of things: A survey," Computer networks, vol. 54, no. 15, pp. 2787-2805, 2010

[3] Z. Dawy, W. Saad, A. Ghosh, J. G. Andrews, and E. Yaacoub, "Towards massive machine type cellular communications," IEEE Wireless Communications Magazine, to appear, 2016.

[4] G. Durisi, T. Koch, and P. Popovski, "Towards massive, ultra-reliable, and low-latency wireless communication with short packets," arXiv preprint arXiv:1504.06526, 2015.

[5] P. Popovski, "Ultra-reliable communication in $5 \mathrm{~g}$ wireless systems," in 1st International Conference on $5 G$ for Ubiquitous Connectivity (5GU), 2014, pp. 146-151.

[6] D. Niyato, X. Lu, P. Wang, D. I. Kim, and Z. Han, "Economics of internet of things: an information market approach," IEEE Wireless Communications, vol. 23, no. 4, pp. 136-145, 2016.

[7] A. P. Athreya and P. Tague, "Network self-organization in the internet of things," in 2013 IEEE International Conference on Sensing, Communications and Networking (SECON), New Orleans, USA, 2013, pp. 25-33.

[8] M. Mozaffari, W. Saad, M. Bennis, and M. Debbah, "Mobile internet of things: Can uavs provide an energy-efficient mobile architecture?" IEEE Global Communications Conference (GLOBECOM), Selected Areas in Communications - Interne of Things Symposium, 2016.

[9] N. Abuzainab, W. Saad, and H. V. Poor, "Cognitive hierarchy theory for heterogeneous uplink multiple access in the internet of things," in IEEE International Symposium on Information Theory (ISIT), Barcelona, Spain, 2016, pp. 1252-1256.

[10] D. Gale and L. Shapley, "College admissions and the stability of marriage," American Mathematical Monthly, pp. 9-15, 1969.

[11] C.-C. Huang and T. Kavitha, "Popular matchings in the stable marriage problem," in International Colloquium on Automata, Languages, and Programming. Springer, 2011, pp. 666-677.

[12] F. Brandl and T. Kavitha, "Popular matchings with multiple partners," arXiv preprint arXiv:1609.07531, 2016.

[13] I. F. Akyildiz, W. Su, Y. Sankarasubramaniam, and E. Cayirci, "Wireless sensor networks: a survey," Computer networks, vol. 38, no. 4, pp. 393-422, 2002.

[14] G. L. Stüber, Principles of mobile communication. Springer Science \& Business Media, 2011
[15] I. F. Akyildiz, M. C. Vuran, and O. B. Akan, "On exploiting spatial and temporal correlation in wireless sensor networks," in Proceedings of WiOpt, vol. 4, 2004, pp. 71-80.

[16] M. Sotomayor, "Two-sided matching: A study in game-theoretic modeling and analysis," Econometric Society Monographs. Cambridge University Press, Cambridge, 1990 .

[17] D. Gale and M. Sotomayor, "Some remarks on the stable matching problem," Discrete Applied Mathematics, vol. 11, no. 3, pp. 223-232, 1985.

[18] T. Kavitha, "A size-popularity tradeoff in the stable marriage problem," SIAM Journal on Computing, vol. 43, no. 1, pp. 52-71, 2014.

[19] T. Kavitha, J. Mestre, and M. Nasre, "Popular mixed matchings," Theoretical Computer Science, vol. 412, no. 24, pp. 2679-2690, 2011.

\section{APPENDIX}

The convergence of the algorithm to the popular outcome guarantees that the players can reach a popular matching in a finite number of iterations. The convergence of the algorithm is guaranteed as the number of players is finite and every device $i \in \mathcal{I}$ applies at most twice from the same SBS. Next, we show that once the algorithm converges, the resulting matching between IoT devices and SBSs is popular.

Based on the popularity of a matching as defined in (16), it is shown in [18] that a matching is popular if it is a complete matching and it has weight at most 0 . Thus, to proof the popularity of the matching derived by Algorithm 1, we show that the matching $\mu$ has weight zero.

First, we define the set $\mathcal{S}^{\prime}$ as a set of SBSs in which for every SBS $s \in \mathcal{S}$ of capacity $q_{s}, q_{s}$ copies of $s$ are created in $\mathcal{S}^{\prime}$. Thus, we have $\mathcal{S}^{\prime}=\left\{s_{k}, 1 \leq k \leq q_{s}, \forall s \in \mathcal{S}\right\}$. To each copy of an SBS $s$, we assign one device $i \in \mu(s)$ from the set of devices that are initially assigned to SBS $s$.

Moreover, a matching is said to be complete if every player is matched in the graph. To make our matching problem complete, every unmatched SBS $s \in \mathcal{S}^{\prime}$ and device $i$ is matched to a virtual player $l(s)$ and $l(i)$, respectively. On the other hand, we denote the SBSs in $\mathcal{S}^{\prime}$ that are matched to devices in $\mathcal{I}_{0}$ as $\mathcal{S}_{0}^{\prime}$ and SBSs that are matched to devices in $\mathcal{I}_{1}$ as $\mathcal{S}_{1}^{\prime}$.

The weight of a matching is defined as follows:

- For an edge $e=\left(i, s_{k}\right) \in \mathcal{I} \times \mathcal{S}^{\prime}$ : The weight of edge $e=$ $\left(i, s_{k}\right)$ is $\mathrm{w}_{\mu}(e)=\mathrm{v}_{i}\left(s_{k}, \mu(i)\right)+\mathrm{v}_{s_{k}}\left(i, \mu^{\prime}\left(s_{k}\right)\right)$. Note that for every edge $e \in \mathcal{I} \times \mathcal{S}^{\prime}$, we have that $\mathrm{w}_{\mu}(e) \in\{ \pm 2,0\}$ and $\mathrm{w}_{\mu}(e)=2$ if and only if $e$ blocks the matching $\mu$.

- For any edge $e=(x, l(x))$ : The weight of edge $e$ is $\mathrm{w}_{\mu}(e)=\mathrm{v}_{x}\left(l(x), \mu^{\prime}(x)\right)$. Thus, $\mathrm{w}_{\mu}(x, l(y))=-1$ if $y$ 
was matched in $\mu$ and $\mathrm{w}_{\mu}(y, h(y))=0$ otherwise (in which case $\mu^{\prime}(y)=l(y)$ ).

To show that max-weight of the matching $\mu$ is 0 , we formulate a primal linear programming (LP) and show that its optimal value is 0 . The primal LP can be defined as follows:

$$
\begin{aligned}
\text { maximize } & \sum_{e \in G_{\mu}} \mathrm{w}_{\mu}(e) \cdot x_{e}, \\
\text { subject to } & \sum_{e \in \mathcal{E}(y)} x_{e}=1 \text { for all } y \in \mathcal{S} \cup \mathcal{I}, \\
& x_{e} \geq 0 \text { for all } e \in G_{\mu},
\end{aligned}
$$

where $\mathcal{E}(y)$ is the set of edges incident on $y$ in $G_{\mu}$ and $x=$ $\left[x_{e}\right]_{\{e \in \mathcal{E}\}} \in[0,1]^{E}$ is the matching polytope and $E$ is the number of edges.

In [19], it is shown that the dual LP of (17) can be given as follows by associating a variable $\kappa_{i}$ for all device $i \in \mathcal{I}$ and $\varphi_{s}$ for all SBS $s \in \mathcal{S}^{\prime}$.

$$
\begin{array}{ll}
\operatorname{minimize} & \sum_{i \in \mathcal{I}} \kappa_{i}+\sum_{s \in \mathcal{S}^{\prime}} \varphi_{s}, \\
\text { subject to } & \kappa_{i}+\varphi_{s} \geq \mathrm{w}_{\mu}(i, s) \text { for all }(i, s) \in G_{\mu}, \\
& \kappa_{i} \geq \mathrm{w}_{\mu}(i, l(i)) \text { for all } i \in \mathcal{I}, \\
& \varphi_{i} \geq \mathrm{w}_{\mu}(s, l(s)) \text { for all } s \in \mathcal{S}^{\prime},
\end{array}
$$

where the values of $\kappa_{i}$ and $\varphi_{s}$ are assigned as follows. For all device $i \in \mathcal{I}$, we set $\kappa_{i}=0$ if device $i$ is unmatched in $\mu$, and for every matched device $i$, we have $\kappa_{i}=1$ if device $i \in \mathcal{I}_{0}$ and $\kappa_{i}=-1$ if device $i \in \mathcal{I}_{1}$. The values of $\varphi_{s}$ are defined similarly. Thus, when SBS $s$ is matched, we have $\varphi_{s}=1$ if $\operatorname{SBS} s \in \mathcal{S}_{1}^{\prime}$, and $\varphi_{s}=0$ if $\operatorname{SBS} s \in \mathcal{S}_{0}^{\prime}$.

Observe that the second and third constraints of (18) hold for all $i \in \mathcal{I}$ and $s \in \mathcal{S}^{\prime}$. This is because $\kappa_{i}=0=\mathrm{w}_{\mu}(i, l(i))$ for all unmatched devices and $\varphi_{s}=0=\mathrm{w}_{\mu}(s, l(s))$ for all unmatched SBSs. Similarly, for all matched devices and SBSs in $\mu$, we have $\kappa_{i} \geq-1=\mathrm{w}_{\mu}(i, l(i))$ and $\varphi_{i} \geq-1=\mathrm{w}_{\mu}(s, l(s))$. In order to show that the first constraint holds, we have the following remark.

Remark 1. Let $(i, s)$ be an edge in $G_{\mu}$.

- If $e=(i, s) \in \mathcal{I}_{1} \times \mathcal{S}_{0}^{\prime}$, then $w_{\mu}(e)=-2$.

- If $e=(i, s) \in\left(\mathcal{I}_{0} \times \mathcal{S}_{0}^{\prime}\right) \cup\left(\mathcal{I}_{1} \times \mathcal{S}_{1}^{\prime}\right)$, then $w_{\mu}(e) \leq 0$.

This result states that given the matching outcome of the algorithm, for every edge $(i, s) \in \mathcal{I}_{1} \times \mathcal{S}_{0}^{\prime}$, we have $\mathrm{w}_{\mu}(e)=$ -2 which means that the two players $s$ and $i$ do not prefer to be matched to one another. Since $\kappa_{i} \geq-1$ and $\kappa_{s} \geq-1$ for all $i \in \mathcal{I}_{1}$ and $s \in \mathcal{S}_{0}^{\prime}$, the first constraint holds for all edges of $G_{\mu}$ in $\mathcal{I}_{1} \times \mathcal{S}_{0}^{\prime}$.

Moreover, given that for every edge $e=(i, s) \in\left(\mathcal{I}_{0} \times\right.$ $\left.\mathcal{S}_{0}^{\prime}\right) \cup\left(\mathcal{I}_{1} \times \mathcal{S}_{1}^{\prime}\right)$, we have $\mathrm{w}_{\mu}(e) \leq 0$ which means that at the end of the algorithm, at least one of the two players $i$ and $s$ do not prefer to be matched to the other player $s$ and $i$, respectively. Then, since $\kappa_{i}+\varphi_{s} \geq 0$ for all $(i, s) \in \mathcal{I}_{k} \times \mathcal{S}_{k}^{\prime}$ for $k \in\{0,1\}$, the first constraint holds for all edges of $G_{\mu}$ in $\left(\mathcal{I}_{0} \times \mathcal{S}_{0}^{\prime}\right) \cup\left(\mathcal{I}_{1} \times \mathcal{S}_{1}^{\prime}\right)$
Now, since $\mathrm{w}_{\mu}(e) \leq 2$ holds for all edges in $G_{\mu}$ and we set $\kappa_{i}=1$ and $\varphi_{s}=1$ for all $i \in \mathcal{I}_{0}$ and $s \in \mathcal{S}_{1}^{\prime}$. Thus, the first inequality holds for all edges in $G_{\mu}$ and thus, $\kappa_{i}$-values and $\varphi_{s}$-values are dual feasible.

For every edge $(i, s) \in \mu$, we have $\kappa_{i}+\varphi_{s}=0, \kappa_{i}=0$, and $\varphi_{s}=0$ for all unmatched devices and SBSs. Hence, it follows that $\sum_{i \in \mathcal{I}} \kappa_{i}+\sum_{s \in \mathcal{S}^{\prime}} \varphi_{s}=0$. So by weak duality, the primal optimal is at most 0 . In other words, the outcome matching of Algorithm 1 that matches all the devices and SBSs has weight at most 0 . 